\title{
The artificial kidney induces AKI? Not if we apply "kidney-protective" renal replacement therapy
}

\author{
M. Legrand ${ }^{1,2^{*}} \mathbb{D}$, J. R. Prowle $e^{3,4,5}$ and L. G. Forni ${ }^{6,7}$
}

(C) 2019 Springer-Verlag GmbH Germany, part of Springer Nature

Undoubtedly, timely and appropriate use of renal replacement therapy (RRT) has saved the lives of many who without such treatment would have succumbed to the consequences of acute kidney injury (AKI). Technological progress together with increased emphasis on multiorgan support has led, in many centres, to implementation of RRT in anticipation of absolute indications being proposed. However, this enthusiasm for "early" treatment has been tempered by investigators critical of this approach who advocate a "less is more" strategy highlighting both costs and the potential adverse effects of inappropriate therapy. Support for this approach is provided by the results of two French randomized controlled trials (AKIKI [1] and IDEAL ICU [2]) which both failed to demonstrate any benefit from earlier implementation of RRT compared to a "delayed" strategy for AKI. Indeed, many patients in the "delayed" group eventually did not receive RRT. Early initiation of RRT in both studies was based on AKI criteria (change in serum creatinine or duration of urine output) in patients with multiple organ failure, and it is no surprise that patients randomized in the more restrictive arm needed less RRT. Potential side effects of unwarranted RRT were further underlined in the AKIKI trial, with a higher incidence of catheterrelated infections in the "early" group. The lack of benefit in these studies has promoted the view that RRT may be harmful and should be employed only in the face of imminently life-threatening consequences of severe renal dysfunction. While we fully agree that applying an

*Correspondence: matthieu.legrand@ucsf.edu

${ }^{1}$ Department of Anesthesia and Peri-operative Care, University of California, San Francisco, CA, USA

Full author information is available at the end of the article invasive therapy when not required is highly undesirable, we feel that the suggestion that RRT is intrinsically harmful and to be avoided at all costs is overly nihilistic and neglects the important role that RRT may place in the integrated management of the patient with multi-organ failure.

Both the mortality and the development of extra-renal organ failure were similar between the early and the delayed group in both the AKIKI and the IDEAL ICU studies. This does not provide support for the hypothesis that RRT is harmful or indeed vice versa. However, what is of interest is that around $50 \%$ of the patients in AKIKI and IDEAL-ICU received intermittent haemodialysis (IHD) as their modality of treatment whilst in the ICU, including many as a sole therapy which differs to contemporaneous practice across the rest of the world. Moreover, approximately $45 \%$ of patients in the IDEALICU study, all of whom had septic shock, receivied IHD as their initial treatment. Concerns have long been raised regarding the risk of additional renal insult resulting from use of IHD in patients with AKI, potentially impairing recovery $[3,4]$. This is likely to be associated with the difficulty in controlling fluid balance with IHD without inducing intravascular hypovolaemia during a time-constrained period of ultrafiltration [5]. Controlling fluid balance over a short period of time (i.e. most often 4-6 h every 2 days) requires high ultrafiltration rates (UF) which induces hypovolemia and haemoconcentration. This phenomenon is observed in the treatment of chronic kidney disease (CKD) and is corrected during the hours following therapy through plasma refilling by the interstitial compartment $[6,7]$. Prescription of inappropriate high doses of UF rate will result in altered systemic hemodynamics [8], decrease cardiac output

\section{Springer}


and induce intra-dialytic hypotension particularly where the individual remains fluid responsive [9]. In a recent study intermittent hemodialysis was shown to decrease renal perfusion to $65 \%$ of baseline in about two-thirds of patients with CKD [10]. The observed decrease in renal perfusion was associated with the intensity of UF $(r=0.31, p=0.05$ with mean UF). Such systemic hemodynamic alternations have long been shown to impair the chance of renal recovery after AKI [11] with more frequent exposure to haemodialysis conferring worse renal outcomes [12].

In AKIKI lower urine output after RRT initiation in the "early" vs restrictive group was reported, an observation interpreted by the authors as an exacerbation of renal injury. Whether under these circumstances oliguria relates to adverse long term renal outcomes is unknown and importantly does this observation reveal an intrinsic side-effect of RRT or is it a consequence of applied modality. Recently, a post hoc analysis of the RENAL trial demonstrated that applying higher UF rates rate during CRRT ( $>1.75 \mathrm{ml} / \mathrm{kg} / \mathrm{h}$ ) was associated with worse outcomes [13]. However, of note, the same authors have previously reported higher UF rates to manage fluid overload were associated with improved outcomes [14]. We believe that overall these observations are consistent with the known strong association between overall fluid balance and adverse outcomes in AKI and the acknowledged difficulty in resolving tissue fluid overload without inducing intravascular hypovolaemia. Seen in this context earlier RRT with a continuous modality might be beneficial in limiting the extent of fluid accumulation, while late therapy, especially with IHD, might be harmful due to the requirement of higher UF rates to resolve accumulated fluid overload in the face of fixed vascular refilling (Fig. 1). Therefore, we believe that the potential for harm arises where therapy is either given for the wrong indication or in an inappropriate fashion or indeed both. By analogy with mechanical ventilation a tidal volume of $6 \mathrm{ml} / \mathrm{kg}$ is less injurious than $12 \mathrm{mkl} / \mathrm{kg}$, however that does not mean that patients with severe respiratory failure do not benefit from some form of mechanical ventilation-just that we have to appreciate and minimize the intrinsic injurious nature. In using RRT, both the modality and UF rate are key factors in development of secondary renal injury. Ultrafiltration during RRT has the ability to "override" the circulation and therefore requires careful monitoring in order to reduce risk of harm. How to reach fluid balance targets needs better understanding of the monitoring and adjustment of UF rates [15].

To conclude, we agree that AKI staging by serum creatinine and/or urine output are insufficient indications for commencing RRT, however RRT remains the key treatment of both the metabolic and fluid overload

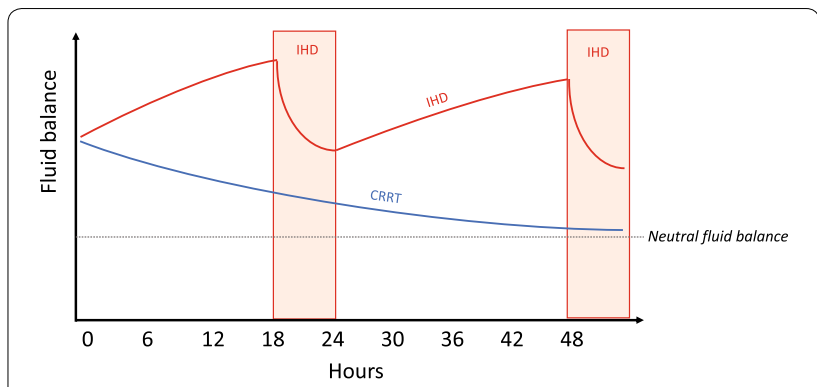

Fig. 1 Representation of fluid balance control using intermittent hemodialysis (IHD) or continuous renal replacement therapy (CRRT) in fluid overloaded patients in the intensive care unit ( adapted from Alex Yartsev, www.derangedphysiology.com)

consequences of severe AKI, in particular in the context of multi-organ dysfunction. To provide safe and effective RRT we need to apply "kidney-protective" therapy as much as possible to limit any perceived harm. In selected patients this may involve earlier implementation of a continuous RRT modality to limit fluid accumulation, while in other contexts watchful waiting may be appropriate. Differentiating these patient groups remains a key challenge in critical care nephrology.

\begin{abstract}
Author details
${ }_{1}^{1}$ Department of Anesthesia and Peri-operative Care, University of California, San Francisco, CA, USA. ${ }^{2}$ F-CRIN INICRCT Network and Inserm U942, Lariboisière, Paris, France. ${ }^{3}$ William Harvey Research Institute, Queen Mary University of London, London, UK. ${ }^{4}$ Adult Critical Care Unit, The Royal London Hospital, Barts Health NHS Trust, Whitechapel Road, London E1 1BB, UK. ${ }^{5}$ Department of Renal Medicine and Transplantation, The Royal London Hospital, Barts Health NHS Trust, Whitechapel Road, London E1 1BB, UK. ${ }^{6}$ Royal Surrey NHS Foundation Trust, Egerton Road, Guildford GU2 7XX, UK. ${ }^{7}$ Department of Clinical and Experimental Medicine, School of Biosciences and Medicine, University of Surrey, Daphne Jackson Rd, Guildford GU2 7WG, UK.
\end{abstract}

\section{Compliance with ethical standards}

\section{Conflicts of interest}

ML received grants from the French Ministry of Health, research support from Sphingotec, lecture fees from Baxter and Fresenius, and consulting fees from Novartis, all outside the submitted work. JP has Consultancy agreements with Medibeacon Inc, Quark Pharmaceuticals Inc, GE Healthcare and Nikkiso Europe $\mathrm{GmbH}$, has received speakers fees travel and hospitality form Baxter Inc, B. Braun Melsungen AG, Nikksio Europe GmbH and Fresenius Medical Care AG. LF received research support from Baxter and Ortho Clinical Diagnostics and consulting fees from La Jolla Pharmaceuticals and Medibeacon.

\section{Ethical approval}

This article does not contain any studies with human participants or animals performed by any of the authors.

\section{Publisher's Note}

Springer Nature remains neutral with regard to jurisdictional claims in published maps and institutional affiliations.

Received: 7 October 2019 Accepted: 9 November 2019 Published online: 20 November 2019 


\section{References}

1. Gaudry S, Hajage D, Schortgen F et al (2016) Initiation strategies for renalreplacement therapy in the intensive care unit. N Engl J Med 375:122133. https://doi.org/10.1056/NEJMoa1603017

2. Barbar SD, Clere-Jehl R, Bourredjem A et al (2018) Timing of renal-replacement therapy in patients with acute kidney injury and sepsis. N Engl J Med 379:1431-1442. https://doi.org/10.1056/NEJMoa1803213

3. Wald R, Shariff SZ, Adhikari NKJ et al (2014) The association between renal replacement therapy modality and long-term outcomes among critically ill adults with acute kidney injury: a retrospective cohort study*. Crit Care Med 42:868-877. https://doi.org/10.1097/CCM.0000000000000042

4. Bonnassieux M, Duclos A, Schneider AG et al (2018) Renal replacement therapy modality in the ICU and renal recovery at hospital discharge. Crit Care Med 46:e102-e110. https://doi.org/10.1097/CCM.000000000000279 6

5. Douvris A, Zeid K, Hiremath S et al (2019) Mechanisms for hemodynamic instability related to renal replacement therapy: a narrative review. Intensive Care Med 45:1333-1346. https://doi.org/10.1007/s00134-019-05707 $-\mathrm{W}$

6. Minutolo R, De Nicola L, Bellizzi V et al (2003) Intra- and post-dialytic changes of haemoglobin concentrations in non-anaemic haemodialysis patients. Nephrol Dial Transplant 18:2606-2612. https://doi.org/10.1093/ ndt/gfg387

7. Saran R, Bragg-Gresham JL, Levin NW et al (2006) Longer treatment time and slower ultrafiltration in hemodialysis: associations with reduced mortality in the DOPPS. Kidney Int 69:1222-1228. https://doi.org/10.1038/ sj.ki.5000186

8. Mahmoud H, Forni LG, Mclntyre CW, Selby NM (2017) Myocardial stunning occurs during intermittent haemodialysis for acute kidney injury. Intensive Care Med 43:942-944. https://doi.org/10.1007/s0013 4-017-4768-2

9. Monnet $X$, Cipriani F, Camous L et al (2016) The passive leg raising test to guide fluid removal in critically ill patients. Ann Intensive Care 6:46. https ://doi.org/10.1186/s13613-016-0149-1

10. Marants R, Qirjazi E, Grant CJ et al (2019) Renal perfusion during hemodialysis: intradialytic blood flow decline and effects of dialysate cooling. J Am Soc Nephrol 30:1086-1095. https://doi.org/10.1681/ASN.2018121194

11. Kelleher SP, Robinette JB, Miller F, Conger JD (1987) Effect of hemorrhagic reduction in blood pressure on recovery from acute renal failure. Kidney Int 31:725-730. https://doi.org/10.1038/ki.1987.58

12. Vijayan A, Delos Santos RB, Li T et al (2018) Effect of frequent dialysis on renal recovery: results from the acute renal failure trial network study. Kidney Int Rep 3:456-463. https://doi.org/10.1016/j.ekir.2017.11.018

13. Murugan R, Kerti SJ, Chang C-CH et al (2019) Association of net ultrafiltration rate with mortality among critically ill adults with acute kidney injury receiving continuous venovenous hemodiafiltration: a secondary analysis of the randomized evaluation of normal vs augmented level (RENAL) of renal replacement therapy trial. JAMA Netw Open 2:e195418. https://doi. org/10.1001/jamanetworkopen.2019.5418

14. Murugan R, Balakumar V, Kerti SJ et al (2018) Net ultrafiltration intensity and mortality in critically ill patients with fluid overload. Crit Care. https:// doi.org/10.1186/s13054-018-2163-1

15. Legrand M, Soussi S, Depret F (2018) Cardiac output and CVP monitoring... to guide fluid removal. Crit Care Lond Engl 22:89. https://doi. org/10.1186/s13054-018-2016-y 